\title{
O FENÔMENO PUZZLE-PUDDLE-PICKLE NA PERSPECTIVA DO MODELO DOS EXEMPLARES
}

\author{
Paloma Maraísa Oliveira Carmo \\ Maria de Fátima de Almeida Baia
}

Submetido em 29 de maio de 2019.

Aceito para publicação em 09 de setembro de 2019.

Cadernos do IL, Porto Alegre, n. ${ }^{\circ}$ 59, outubro. p. 125-148.

\section{POLÍTICA DE DIREITO AUTORAL}

Autores que publicam nesta revista concordam com os seguintes termos:

(a) Os autores mantêm os direitos autorais e concedem à revista o direito de primeira publicação, com o trabalho simultaneamente licenciado sob a Creative Commons Attribution License, permitindo o compartilhamento do trabalho com reconhecimento da autoria do trabalho e publicação inicial nesta revista.

(b) Os autores têm autorização para assumir contratos adicionais separadamente, para distribuição não exclusiva da versão do trabalho publicada nesta revista (ex.: publicar em repositório institucional ou como capítulo de livro), com reconhecimento de autoria e publicação inicial nesta revista.

(c) Os autores têm permissão e são estimulados a publicar e distribuir seu trabalho online (ex.: em repositórios institucionais ou na sua página pessoal) a qualquer ponto antes ou durante o processo editorial, já que isso pode gerar alterações produtivas, bem como aumentar o impacto e a citação do trabalho publicado.

(d) Os autores estão conscientes de que a revista não se responsabiliza pela solicitação ou pelo pagamento de direitos autorais referentes às imagens incorporadas ao artigo. A obtenção de autorização para a publicação de imagens, de autoria do próprio autor do artigo ou de terceiros, é de responsabilidade do autor. Por esta razão, para todos os artigos que contenham imagens, o autor deve ter uma autorização do uso da imagem, sem qualquer ônus financeiro para os Cadernos do IL.

\section{POLÍTICA DE ACESSO LIVRE}

Esta revista oferece acesso livre imediato ao seu conteúdo, seguindo o princípio de que disponibilizar gratuitamente o conhecimento científico ao público proporciona sua democratização.

http://seer.ufrgs.br/cadernosdoil/index

Terça-feira, 29 de outubro de 2019. 


\title{
O FENÔMENO PUZZLE-PUDDLE-PICKLE NA PERSPECTIVA DO MODELO DOS EXEMPLARES
}

\author{
THE PUZZLE-PUDDLE-PICKLE PHENOMENON \\ BASED ON THE PERSPECTIVE OF THE EXEMPLARS \\ MODEL
}

\author{
Paloma Maraísa Oliveira Carmo* \\ Maria de Fátima de Almeida Baia ${ }^{* *}$
}

\begin{abstract}
RESUMO: Neste estudo, apresentamos e discutimos o paradigma dos Sistemas Adaptativos Complexos (THELEN; SMITH, 1994; LARSEN-FREEMAN, 1997) com o modelo dos exemplares (BYBEE, 2001, 2016; CRISTÓFARO-SILVA, 2003) como uma abordagem alternativa para explicar o fenômeno puzzlepuddle-pickle (PPP), caracterizado por contextos em que a criança se mostra capaz de produzir determinado segmento, mas falha em outro. Para isso, analisamos tokens de uma criança que desenvolve o português brasileiro entre 1 e 2 anos. Após análise dos dados, mostramos que se trata de um fenômeno de baixa frequência e que não tem relação com templates operantes, i.e rotinas articulatórias iniciais. Por essa razão, o modelo multirrepresentacional dos exemplares, que leva em consideração aspectos de diferentes componentes linguísticos e extralinguísticos, apresenta vantagens na sua abordagem.
\end{abstract}

PALAVRAS-CHAVE: puzzle-puddle-pickle; sistemas adaptativos complexos; modelo dos exemplares.

ABSTRACT: In this study, we present and discuss the paradigm of Complex Adaptive Systems (THELEN; SMITH, 1994; LARSEN-FREEMAN, 1997) with the Exemplars model (BYBEE, 2001, 2016; CRISTÓFARO-SILVA, 2003) as an alternative approach to explain the Puzzle-Puddle-Pickle phenomenon (PPP), characterised by contexts in which the child is able to produce a particular segment, but fails in another. We analyse tokens of a child who develops Brazilian Portuguese from 1 to 2 years. After analysing the data, we show that it is a low frequency phenomenon which has no relation with templates, i.e. initial articulatory routines. For this reason, the multi-representational Model of Exemplars has advantages in its approach as it takes into account aspects of different linguistic and extralinguistic components.

KEYWORDS: Puzzle-Puddle-Pickle; adaptive complex systems; exemplars model.

\section{Introdução}

Neste estudo, discutimos e analisamos a relação do léxico inicial com o desenvolvimento fonológico nos dados de uma criança. Em específico, investigamos o fenômeno puzzle-puddle-pickle no desenvolvimento do português brasileiro (PB) da variedade de Vitória da Conquista-BA. Para tanto, é assumido o quadro teórico dos Sistemas Adaptativos Complexos (SACs) (THELEN; SMITH, 1994), que assume

\footnotetext{
* Mestranda pelo Programa de Pós-Graduação em Linguística (PPGLIN) da Universidade Estadual do Sudoeste da Bahia (UESB), bolsista CAPES, palomamaraisa@ gmail.com.

** Professora doutora no Programa de Pós-Graduação em Linguística (PPGLIN) e no Departamento de Estudos Linguísticos e Literários (DELL) da Universidade Estadual do Sudoeste da Bahia (UESB), mariadefatimabaia@uesb.edu.br.
} 
variabilidade, instabilidade e não linearidade no sistema em desenvolvimento. Nessa perspectiva, o sistema não segue uma ordem pré-estipulada, por estar aberto à entrada de energia para que mudanças ocorram durante o desenvolvimento e padrões possam emergir de forma variável e dinâmica. Em consonância com os SACs, é assumido, ainda, o modelo multirrepresentacional dos exemplares (PIERREHUMBERT, 2012; BYBEE, 2001, 2002, 2008; CRISTÓFARO-SILVA, 2003; OLIVEIRA-GUIMARÃES, 2008), que capta essa variabilidade e instabilidade no percurso de desenvolvimento fonológico infantil a partir de sobreposição de exemplares em competição no sistema em desenvolvimento.

Em geral, a perspectiva dos SACs (THELEN; SMITH, 1994; LARSENFREEMAN, 1997; DE BOT et al, 2007), vinculada ao modelo dos exemplares (PIERREHUMBERT，2012; BYBEE，2001，2008，2016; CRISTÓFARO-SILVA, 2003), defende que o desenvolvimento da linguagem inicial é dependente da interação entre múltiplos elementos, e a experiência tem um impacto na representação fonológica. Nesse sentido, a criança, no período inicial de desenvolvimento linguístico, é capaz de adquirir novos padrões a partir do seu contexto linguístico e da associação entre elementos já existentes que emparelham forma e significado. A experiência e a frequência, portanto, são fundamentais para a representação linguística. Todavia, como Pierrehumbert (2012) observa, o fato de ser possível a aplicação dos SACs nos estudos da linguagem não pressupõe que todo sistema dinâmico funcione com uma gramática. Para a autora, qualquer teoria linguística que se baseie na perspectiva dinâmica precisa ser capaz de reproduzir e explicar as regularidades da gramática. Essa necessidade tem sido um dos maiores desafios dos estudos de linguagem recentes que fazem uso dos Sistemas Dinâmicos.

No que se refere à relação entre o léxico e os padrões fônicos iniciais, segundo Gerken (2008), o léxico compreende um conjunto de palavras partilhadas e internalizadas pelo falante em uma dada comunidade linguística a partir de um acervo vocabular. Em termos de desenvolvimento, podemos afirmar que a entrada lexical é um processo complexo e contínuo que, além disso, ocorre em correspondência com outros subsistemas. Em uma visão dinâmica, Pierrehumbert (2012), ao abordar a relação entre léxico e fonologia, defende um "léxico dinâmico" que seria o locus central da associação entre forma e significado e o armazenamento abstrato (fonológico) e detalhado (fonético) das palavras.

De acordo com Brum-de-Paula e Ferreira-Gonçalves (2008), a constituição do léxico, pensando no desenvolvimento linguístico, resulta da interação entre vários domínios da linguagem. Assim, o desenvolvimento lexical é determinado pelo inventário fonológico da criança e vice-versa, o que permite considerar que as primeiras palavras já expressam a emergência da representação fonológica de modo dinâmico, não linear e rico em detalhamento fonético.

As representações linguísticas se formam por meio de uma memória enriquecida, uma vez que são armazenadas todas as informações que um falante pode extrair da sua experiência linguística. Dessa maneira, o modelo dos exemplares tem sido usado nos estudos dos SACs como meio de explicar a relação entre estrutura linguística, léxico e significado. O uso desse modelo é pertinente para a perspectiva dinâmica devido ao seu caráter multirrepresentacional. De acordo com Cristófaro-Silva e Oliveira-Guimarães (2011), os modelos multirrepresentacionais assumem a representação linguística do componente fonológico como multiplamente especificada, 
por incluir fonemas, alofones e detalhes fonéticos, além de informações extralinguísticas.

Ademais, a fim de demonstrar a relação do léxico inicial com a representação fonológica, seguimos o modelo dos exemplares (PIERREHUMBERT, BYBEE, 2001; CRISTÓFARO-SILVA; 2003; OLIVEIRA-GUIMARÃES, 2008) no intuito de apresentar uma explicação alternativa para o fenômeno puzzle-pudlle-pickle (doravante PPP) (MACKEN, 2008), que ilustra a não estabilização de um segmento em uma determinada palavra, mas que é realizado em outra.

Delimitamos uma questão para nortear este trabalho: haveria relação entre o léxico inicial e o desenvolvimento fonológico, em específico, com os templates? Como reportado na literatura (BRUM-DE-PAULA; FERREIRA-GONÇALVES, 2008; WIETHAN et al., 2014), o desenvolvimento lexical e o fonológico estão interrelacionados. Ou seja, o inventário lexical das crianças acomoda detalhes fônicos que influenciam na emergência de novos itens e vice-versa. Essa questão refere-se à hipótese deste trabalho: o desenvolvimento fonológico não opera isoladamente, há uma estreita relação entre os padrões fônicos emergentes e outros aspectos presentes.

\section{Desenvolvimento fonológico e lexical: explicação com base no modelo dos exemplares}

O modelo dos exemplares (SMITH, 1981; BYBEE, 2001; CRISTÓFAROSILVA, 2003; MATLIN, 2004; BOD; COCHRAN, 2007; OLIVEIRA-GUIMARÃES, 2008) foi introduzido, na Psicologia, como modelo para explicar a formação de categorias. Recentemente, nos estudos linguísticos, tem sido mais explorado pela fonética e pela fonologia como uma proposta alternativa para explicar as representações mentais e, também, explicar o funcionamento e a categorização das unidades linguísticas.

É importante enfatizar, ainda, que não se trata de um modelo específico para a linguagem. Na verdade, trata-se de um modelo capaz de explicar processos de armazenamento na memória de domínio geral, que compreende processos operantes em outras áreas da cognição humana, os quais não são, estritamente, linguísticos. Em virtude dessa complexidade, as representações, com base em um modelo de exemplar, têm uma organização rica e detalhada por incluírem tanto aspectos linguísticos quanto aspectos extralinguísticos.

Segundo o modelo, os exemplares representam um conjunto de itens lexicais experienciados pelo falante. Esses exemplares são organizados em redes de generalizações que conectam abstrações em vários níveis, isto é, o falante estoca todas as formas possíveis atestadas em sua experiência por meio de esquemas que expressam generalizações. Além disso, o modelo dos exemplares é entendido como um modelo de representação de memória que, vinculado à Fonologia de Uso, considera níveis de abstração por meio da categorização de exemplares armazenados a partir de similaridades fonéticas, semânticas e do contexto de uso. Nessa perspectiva, as representações linguísticas consistem em uma memória enriquecida, uma vez que são armazenadas todas as informações que um falante pode extrair da sua experiência linguística.

Segundo Cristófaro-Silva e Oliveira-Guimarães (2011), os modelos multirrepresentacionais assumem a representação linguística do componente fonológico 
como multiplamente especificada, incluindo fonemas, alofones e detalhes fonéticos finos, por exemplo, além de informações sobre o falante e o contexto pragmático no qual a palavra está contextualizada.

A experiência, de acordo com o modelo dos exemplares, tem impacto na representação da linguagem (BYBEE; CACOULLOS, 2008; BYBEE, 2016), e a frequência das unidades linguísticas tem um efeito na organização fonológica inicial, i.e. no mapeamento fonológico. Aplicada aos estudos da linguagem, a experiência, juntamente com a frequência, favorece os processos de: a) convencionalização, b) associação, e c) automatização das unidades linguísticas, que assumem comportamentos variáveis, gradientes e emergentes. A convencionalização trata-se da estabilização de um exemplar na memória do indivíduo a partir da frequência de uso, tornando-se mais produtivo. A associação permite que o indivíduo associe formas fonéticas, semânticas e pragmáticas a elementos já experienciados. Por fim, a automatização permite que as unidades associadas se relacionem de maneira convencional, possibilitando a produção automática dos elementos.

Uma vez que as unidades linguísticas armazenadas influenciam na categorização de novos itens, o processo de associação é ativado na memória do indivíduo. Haja vista essa associação, as unidades são mapeadas de acordo com a similaridade dos exemplares já estocados (BOD; COCHRAN, 2007), i.e. as palavras que apresentam significados semelhantes são alocadas próximas umas das outras e, quando um exemplar é acessado, elas ativam, automaticamente, outros itens similares. Cada ocorrência de uso reforça a representação de exemplares que vão se sobrepondo e se tornando mais fortes nas nuvens; por conseguinte, são mais produtivos e mais prováveis de serem ativados na produção. O uso contínuo dessas categorias favorece, automaticamente, a convencionalização no léxico do indivíduo.

Ao tratar da organização fonológica, Bybee (2016) descreve os processos gerais inerentes à organização da seguinte maneira:

a) Categorização: refere-se à similaridade ou ao emparelhamento de unidades que ocorrem quando palavras ou construções são reconhecidas e associadas a categorias já mapeadas na memória do indivíduo. As categorias resultantes, nesse processo, constituem a base do sistema linguístico;

b) Chunking: nesse processo, as construções partem da união de um conjunto de chunks já formados na memória do indivíduo e fundidos em uma só unidade. Desse modo, as sequências de unidades repetidas são agrupadas juntas para serem acessadas como uma unidade simples;

c) Memória enriquecida: trata-se do armazenamento de categorias linguísticas e não linguísticas. Uma representação enriquecida inclui detalhes fonéticos para palavras e construções mais complexas, bem como informações contextuais e semânticas. Isso implica uma representação redundante de informações já experienciadas e mapeadas em nuvens de exemplares;

d) Analogia: esse processo se refere à formação de novas categorias a partir de unidades previamente experienciadas, levando em consideração a forma e o significado.

É fundamental salientar que esses processos estão inter-relacionados e operam, paralelamente, na representação linguística. Bybee (2016) defende que os exemplares exibem um efeito prototípico, tendo em vista uma organização gradual a partir do nível 
de experiência. Isso significa dizer que, na visão da autora, os exemplares apresentam membros mais centrais, caso sejam mais produtivos. No entanto, estudos de Psicologia Cognitiva questionam se de fato há um elemento considerado mais central na representação de exemplares (MATLIN, 2004).

Além de Matlin (2004), há outros estudos que defendem que os protótipos não são equivalentes a exemplares (ALTOM; MEDIN; MURPHY, 1984). Segundo Matlin (2004), esses modelos são avaliados separadamente. As razões para isso são: a) primeiro, a abordagem de protótipos sugere que as categorias levem em consideração somente o item que for mais típico, reduzindo a totalidade de informações em meio a um conjunto de variedades em uma única abstração; segundo, as categorias têm uma estrutura gradual, partindo dos membros mais representativos, ou prototípicos, para os menos prototípicos; b) no modelo dos exemplares, são registradas todas as ocorrências sem uma hierarquia na representação; essas categorias são armazenadas à medida que são usadas, sobrepondo-se a cada uso, sejam elas mais típicas ou não.

Nas palavras de Altom, Medin e Murphy (1984, p. 334, tradução nossa), “[...] a categorização dos modelos de exemplares difere muito dos modelos de protótipos, já que a avaliação de categorias se baseia na recuperação de informações sobre membros de categorias específicas e não em informações resumidas sobre atributos típicos"1. Dessa maneira, na visão dos exemplares, os conceitos são representados por um conjunto variável de categorias, inclusive redundantes, e não por uma representação ordenada linearmente, isto é, ao pensar na representação de exemplares, devem ser levados em consideração vários elementos sobrepostos à medida que são acessados pelo indivíduo em detrimento de uma representação hierárquica ou prototípica.

Nesse sentido, em um modelo dos exemplares, as representações linguísticas são amplas e redundantes e, além disso, exibem gradiência e variação (LANGACKER, 2000; BYBEE, 2002, 2016). Em termos de desenvolvimento linguístico inicial, cada novo exemplar tem um impacto na representação, e as mudanças que ocorrem são implementadas à medida que seu uso se torna mais frequente ao longo do tempo.

Assim, nessa perspectiva, as estruturas linguísticas não se encontram previamente estocadas, mas são adquiridas conforme a exposição e a generalização de exemplares que são alocados em espaços semânticos, contextuais e formas semelhantes. A respeito disso, Oliveira-Guimarães (2008) argumenta que:

\begin{abstract}
Ao ouvir uma determinada forma de uma palavra, o falante/ouvinte a compara com os exemplares já existentes dessa mesma categoria e a armazena junto com os demais membros, constituindo uma rede de relações, a qual se organiza a partir de parâmetros como: frequência, similaridade (fonética, semântica, morfológica, contextual) e robustez. Ou seja, há sobreposição de formas distintas de uma mesma palavra. As formas que são mais (sic) frequentes têm uma representação mais robusta no feixe daquela categoria (OLIVEIRA-GUIMAR ̃̃ES, 2008, p. 82).
\end{abstract}

É sabido, ainda, que os exemplares podem ser formados por diferentes categorias em diferentes níveis de complexidade: tanto abaixo do nível da palavra, que correspondem às unidades fonéticas, quanto em construções mais complexas preenchidas por sintagmas. É fundamental enfatizarmos que essas categorias são

\footnotetext{
1 “"...] exemplar models of categorization differ sharply from the prototype models in their assumption that category judgments are based on retrieval of information about specific category members rather than on summary information about typical attributes".
} 
ativadas a partir da experiência que o falante tem da língua. No que se refere ao armazenamento dos itens, Pierrehumbert (2003) explica que as palavras são armazenadas em grupos que apresentam características fonotáticas e prosódicas similares. Além disso, na perspectiva dinâmica seguida pela autora, essas representações linguísticas não são estáticas, pois podem ser modificadas de acordo com algum tipo de interferência.

Dado que o uso contínuo de categorias contribui para o desenvolvimento lexical, a frequência assume um papel determinante no mapeamento linguístico e no funcionamento das categorias. Todas as unidades experienciadas são registradas na memória, a partir das similaridades, e acomodam todas as possibilidades de realização. Categorias mais frequentes estão mais aptas a apresentarem um maior número de exemplares, ao passo que categorias menos frequentes tendem a diminuir o registro de exemplares categorizados. Essas categorias são mapeadas em forma de nuvens de exemplares compostas por informações linguísticas e não linguísticas, como a figura a seguir apresenta.

Fonte: CRISTÓFARO-SILVA, 2003.

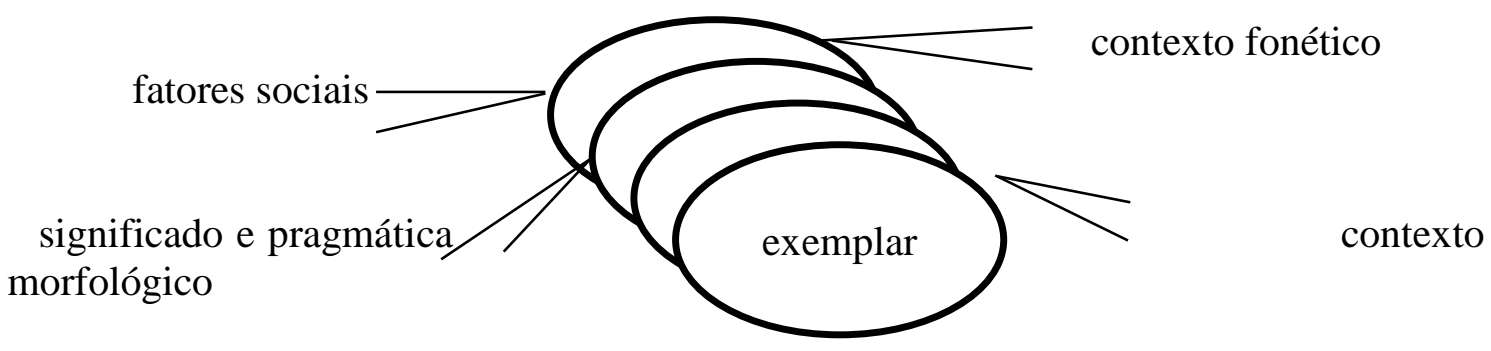

Figura 1 - Nuvem de exemplares

Conforme os exemplares são acessados ou atualizados, eles vão ganhando força no léxico do indivíduo, sobrepondo-se nas nuvens de exemplares. Em contrapartida, aqueles que não forem reforçados tendem a desaparecer da memória do falante. Segundo Bybee (2008),

[...] para cada palavra ou frase no léxico de um falante, há uma nuvem ou conjunto de exemplares fonéticos. O significado da palavra ou frase também é representado por nuvens de exemplares que representam o contexto e o significado de cada símbolo de uma palavra. Propõe-se que a memória para objetos linguísticos seja a mesma para objetos não-linguísticos, o que significa que essas memórias também podem decair (BYBEE, 2008, p. 400, tradução nossa) $)^{2}$

Além disso, as palavras mais frequentes apresentam mais variação e mais mudança em uma velocidade maior em relação às que apresentam baixo número de

\footnotetext{
2 "Thus for every word or phrase in a speaker's lexicon, there is a cloud or cluster of phonetic exemplars. The meaning of the word or phrase is also represented by clusters of exemplars which represent the context and meaning for each token of a word. It is proposed that memory for linguistic objects is the same as for non-linguistic objects, which means that memories can also decay".
} 
ocorrência. Bybee (2016) apresenta duas explicações no que tange ao efeito dessa frequência: primeiro, exemplares mais frequentes são mais fáceis de acessar em tarefas de escolha lexical; em segundo, palavras de alta frequência apresentam maior estabilidade. Ou seja, as palavras mais frequentes apresentam uma maior autonomia lexical.

Apesar de os itens mais frequentes serem mais acessíveis e estocados em uma velocidade superior aos itens menos frequentes, isso não significa que as categorias que ocorrem em menor escala não sejam armazenadas. Pelo contrário, as unidades que ocorrem em menor frequência são mapeadas a partir do controle de co-ocorrência na experiência. Nesse caso, os falantes apresentam exemplares em competição que estão representados na sua comunidade de fala.

Dessa maneira, a representação fonológica, baseada no modelo dos exemplares, é gerenciada pela força lexical com que as categorias tendem a ocorrer no sistema, podendo ser excluídas ou não da representação mental. Assim, quando a repetição é contínua, as categorias são reforçadas no léxico, exibem um número maior de tokens e ganham mais força lexical, ao passo que exemplares mais fracos ou não repetidos as perdem e são excluídos da representação mental. É a frequência, nesse caso, que determina essa categorização. Segundo Bybee (2008), os efeitos da frequência são incorporados nesse modelo de três modos:

\begin{abstract}
1) Os exemplares são reforçados com cada uso, tornando-se mais propensos a serem escolhidos para uso subsequente, 2) palavras de alta frequência são susceptíveis de ter um maior intervalo de variação de modo que sua nuvem de exemplares será maior, e 3) cada uso de um exemplar em tempo real tem o potencial de ter efeitos fonéticos impactando sobre ele. É o terceiro ponto que fornece o mecanismo para os efeitos fonéticos maiores em palavras de alta frequência (BYBEE, 2008, p. 402, tradução nossa). ${ }^{3}$
\end{abstract}

A cada uso, as categorias são movidas, gradualmente, para a parte superior e são mapeadas em forma de nuvens de exemplares, afetando os tokens já experienciados. Em um modelo dos exemplares, todas as ocorrências são registradas na memória. Sendo assim, de acordo com Bybee (2013), não há limites para a quantidade de categorias a serem armazenadas na memória do indivíduo, visto que a capacidade neural é muito maior do que se imagina.

Em suma, a frequência afeta a natureza da representação mental, visto que palavras frequentemente acessadas têm uma força lexical maior em relação às de baixa frequência. A respeito da frequência, há de se considerar duas maneiras distintas de avaliá-la no mapeamento de exemplares: a frequência de token e frequência de type.

A frequência de token é designadamente o número total de ocorrências de uma palavra ou de uma construção particular. Segundo Vigário, Frota e Martins (2010), esse tipo de frequência tem algumas implicações:

[...] acentua a importância da distribuição de unidades e padrões efetivamente presentes no input, que varia de língua para língua [...] acentua também a relevância do uso da língua e do estudo dos possíveis diferentes inputs numa

\footnotetext{
3 “1) Exemplars are strengthened with each use, making them more likely to be chosen for subsequent use, 2) high frequency words are likely to have a greater range of variation so that their exemplar clusters will be larger, and 3) each use of an exemplar in real time has the potential for phonetic effects to have an impact on it. It is the third point that provides the mechanism for the greater phonetic effects on high frequency words".
} 
mesma língua a que diferentes crianças possam estar expostas (VIGÁRIO; FROTA; MARTINS, 2010, p. 764).

Ademais, uma construção de token é estabelecida por um conjunto de itens que ocorrem em cada posição (fonética, morfológica, sintática, pragmática), formando novas categorias (BYBEE, 2013). Nesse caso, há uma contagem geral de todas as categorias formadas, inclusive as repetidas. Oliveira-Guimarães (2008) apresenta um exemplo de token 'st' em uma palavra como pista que ocorreu dez vezes. Nesse caso, todas as ocorrências da palavra com a sequência 'st' foram computadas.

A frequência de token exibe, ainda, um efeito potencial na representação linguística: palavras de alta frequência têm uma taxa maior de mudança do que palavras menos frequentes (BYBEE, 2001). Tratam-se das mudanças fonéticas que progridem mais rapidamente em itens com alta frequência. Isso é notório nas construções em que palavras ou sintagmas sofrem redução a partir de processos fonéticos aplicados em tempo real nas palavras em uso.

Já a frequência de type se refere a uma frequência dicionarizada de padrões particulares, ou seja, listagem de palavras únicas (VIGÁRIO; FROTA; MARTINS, 2010). Esse tipo de frequência é avaliado no contexto de construção e o seu efeito está relacionado à produtividade que corresponde à probabilidade de uma construção ser aplicada a outros itens. A título de exemplificação, Bybee (2016) apresenta o seguinte: na construção "drive someone crazy" ("deixar alguém maluco"), a posição do verbo pode ser preenchida pelos verbos "drive" ("deixar"), "send" ("mandar, enviar"), "make" ("fazer"), ao passo que a do adjetivo pode ser ocupada por uma variedade de tipos, tornando-se, dessa maneira, a construção mais produtiva.

De modo geral, tanto a frequência de token quanto a frequência de type são importantes para compreender como as categorias podem afetar a formação de palavras e construções que estão, constantemente, em mudança. Essas mudanças resultam do uso da língua e o seu efeito de frequência e repetição. Pensando nos exemplares, em consonância com a perspectiva dos SACs, essa mudança é fundamental para as representações cognitivas da linguagem bem como para a formação de padrões linguísticos, uma vez que o indivíduo passa por diferentes momentos de instabilidade até atingir a estabilidade. Cada mudança exibida no sistema do indivíduo provoca um efeito na sua própria gramática.

\section{Primeiras palavras: o fenômeno puzzle-puddle-pickle}

Quando a criança está adquirindo as primeiras palavras, ela se apropria de estratégias para a organização do seu sistema fonológico. Essas estratégias incluem, em geral, exploração dos sons favoritos, exploração da reduplicação ou produção monossilábica, redução de sílabas ou segmentos etc. (VIHMAN, 1993). Em certa medida, esses meios utilizados pelas crianças podem resultar em adaptações/trocas fonológicas no interior da palavra cujo propósito é se aproximar da forma-alvo.

Essas trocas fonológicas ocorrem quando as crianças produzem um determinando segmento em um modo ou ponto de articulação, mas em um momento posterior, podem não realizá-lo. Segundo Macken (2008),

O exemplo mais interessante das muitas-muitas correspondências... é fornecido pelo fenômeno dos "puzzles". Isto é, a criança parece incapaz de 
produzir um som particular ou sequência de som no lugar correto, mas é perfeitamente capaz de produzir como sua interpretação de outra coisa. Por exemplo, por regras completamente regulares puddle foi pronunciado [pgəl] enquanto puzzle foi pronunciado [pdəl]. Ou seja, temos a matriz /pzəl/ [pdəl]; /pdəl/ [pdəl] (MACKEN, 2008, p. 6, tradução nossa). ${ }^{4}$

O fenômeno puzzle-puddle-pickle (PPP) ilustra a não estabilização de um segmento no interior de uma palavra, que, segundo Bybee (2001), é substancialmente mais rica do que o segmento isolado. Em outras palavras, trata-se de um fenômeno caracterizado por contextos em que a criança se mostra capaz de produzir determinado segmento, mas falha em outro. Lamprecht et al (2004) apresentam um exemplo do fenômeno no desenvolvimento do PB, nele a criança produz 'sala' como [tala] e 'chave' como [savi].

Com base em uma explicação estruturalista, a criança, ao trocar um segmento pelo outro, demonstraria que ainda não adquiriu todos os fonemas previstos em sua língua materna. Para Jakobson (1972), o desenvolvimento dos segmentos se daria por meio de feixe de traços lineares. Além disso, haveria uma cronologia na aquisição desses segmentos, razão pela qual a criança estaria trocando um ' $t$ ' por um ' $k$ ', por exemplo. No entanto, como a perspectiva de Jakobson explicaria o fato de a troca acontecer em um grupo de palavras e não em outro?

Estudos derivacionais explicam esse fenômeno com base em uma representação subjacente de generalizações de padrões sonoros com regra ordenada ou armazenada de maneira errônea (SMITH, 1963). Por outro lado, estudos com base em restrições, como os da Teoria da Otimidade (TO), explicam que no estágio inicial de desenvolvimento haveria um ordenamento específico e diferente das restrições da gramática adulta (DINNSENN; O'CONNOR; GIERUT, 2001; SCHWINDT, 2010). Nesse ponto de vista, durante o desenvolvimento linguístico, a criança apresentaria diferentes hierarquias, cada qual refletindo um estágio de seu desenvolvimento. Em geral, nos estudos de aquisição da linguagem com base na TO, a fala infantil é frequentemente não marcada quando comparada com a língua a ser adquirida. Em TO isso pode ser expresso assumindo que as restrições vêm, inicialmente, ranqueadas de acordo com as restrições estruturais, as quais estão, assim, ranqueadas acima das restrições de fidelidade (MCCARTHY; PRINCE, 1993; LEVELT et al, 2000).

Em uma perspectiva dos SACs e de uso, esse fenômeno seria resultado de momentos de instabilidade e variabilidade no percurso de aquisição infantil. Os momentos são gerenciados por uma reorganização interna devido ao princípio da autoorganização, que consiste na formação espontânea de padrões, para atingir uma ordem em um determinado ponto.

Por ser armazenado um exemplar (palavra) com determinado segmento em um ponto ou modo de articulação e estar ausente em outra, característica do fenômeno PPP, podemos afirmar que a representação inicial do desenvolvimento fonológico seria a palavra (cf. VIHMAN; CROFT, 2007). Assim, durante o desenvolvimento, a criança não aprenderia segmentos isolados, pois, na verdade, a palavra seria o locus de categorização.

\footnotetext{
4 "[...] the most interesting instance of the many-many correspondences... is provided by the phenomenon of 'puzzles'. That is, the child appears unable to produce a particular sound or sound sequence in the correct place, but is perfectly capable or producing it as his interpretation of something else. For instance, by completely regular rules puddle was pronunced $[\mathrm{p} \wedge \mathrm{g}$ ol] whilst puzzle was pronounced $[\mathrm{p} \wedge \mathrm{d} ə \mathrm{l}]$. That is, we have the array $/ \mathrm{p} \wedge \mathrm{zəl} / \rightarrow[\mathrm{p} \wedge \mathrm{d} ə \mathrm{l}] ; / \mathrm{p} \wedge \mathrm{d} ə \mathrm{l} / \rightarrow[\mathrm{p} \wedge \mathrm{dəl} / "$.
} 
Por essa razão, este estudo se apoia em uma perspectiva holística, na qual a palavra é tomada como a unidade emergencial do desenvolvimento fonológico. Para Bybee (2001), as palavras são unidades de uso tanto fonológico quanto pragmático. Elas podem ser categorizadas via percepção e produção e a sua identificação não é uma tarefa simples e fácil, uma vez que é difícil precisar o que vem a ser uma palavra. Ademais, o que Gerken (2008), por exemplo, apresenta como protopalavra, ou seja, produção que se distancia do alvo e, em geral, formada por duas sílabas, pode ser interpretada como palavra na perspectiva da Whole-Word Phonology (VIHMAN; CROFT, 2007), caso apresente evidências de manifestação de um template ${ }^{5}$ predominante.

Seguindo essa proposta, a palavra seria definida a partir da aplicação de padrões durante o gerenciamento do conhecimento linguístico. Nas palavras de Cristófaro-Silva e Oliveira-Guimarães (2011), a palavra assume papel fundamental como locus organizador da sonoridade para acomodar padrões que as crianças buscam construir. Devemos enfatizar, ainda, que apesar de muitas formas infantis não apresentarem relação com o alvo, o seu significado pode ser recuperado pelo contexto específico.

Salientamos que a aplicação de um padrão na representação fonológica inicial pode ser considerada um indício de que o infante dispõe de uma representação holística, i.e a palavra. Essa proposta holística defende que a criança não aprende segmentos isolados, mas categorias sonoras acopladas na palavra inicial bem como determinados padrões que facilitam a expansão lexical.

Ademais, é fundamental enfatizar que a ocorrência do fenômeno PPP é diferenciada entre as crianças, que apresentam um comportamento verbal variável, dinâmico e idiossincrático, além de possuírem estruturas fonotáticas próprias do sistema alvo.

\section{Metodologia}

Os dados discutidos nesta pesquisa são dados observacionais e longitudinais, provenientes de fala espontânea de uma criança com desenvolvimento típico: uma criança do sexo feminino, nomeada como L. A criança é residente na cidade baiana de Vitória da Conquista e seus dados são pertencentes ao banco de dados do Grupo de Estudos de Psicolinguística e Desenvolvimento Fonológico (GEPDEF) ${ }^{6}$. Por se tratar de estudo longitudinal, são analisadas sessões com intervalos mensais, no período de $1 ; 0$ a 2;0 anos, com duração de cerca de 30 minutos cada uma, em contextos espontâneos de fala entre a criança, cuidadores e pesquisadores.

Durante as sessões, foram realizadas diferentes brincadeiras, contações de histórias, canções infantis, visando à estimulação de fala das crianças. Após as gravações, os vídeos foram transferidos da câmera digital e da câmera do computador para um HD externo. As gravações em vídeo foram realizadas com filmadora da marca Samsung modelo PL60. Coletados os vídeos, os dados foram transcritos baseando-se no formato CHAT de transcrição da plataforma CHILDES.

\footnotetext{
${ }^{5}$ Templates são rotinas articulatórias usadas de maneira sistemática para a expansão do léxico.

6 Coleta de dados aprovada pelo comitê de ética do projeto maior "Padrões emergentes no desenvolvimento fonológico típico e atípico" (CAAE 30366814.1.0000.0055), coordenado pela Profa. Maria de Fátima de Almeida Baia.
} 
Na tabela 1, a seguir, é apresentado o total de produções realizadas pela criança e analisadas neste estudo.

Tabela 1 - Total de produções de palavras de L.

\begin{tabular}{c|ll}
\hline Criança & \multicolumn{1}{c}{ Faixa etária } & $\begin{array}{l}\text { Total de produções de } \\
\text { palavras }\end{array}$ \\
\hline 1. L. & $\begin{array}{l}1 ; 0-2 ; 0 \\
13 \text { sessões/meses }\end{array}$ & 1256 tokens \\
\hline
\end{tabular}

Por serem dados iniciais, para categorizar produções como palavra e não balbucio, foram considerados os critérios elaborados por Vihman e MacCune (1994), que são aplicados em palavras que geram dúvidas quanto à categorização. Quando há candidatos que geram dúvidas, é preciso considerar aspectos fônicos relacionados ao alvo e/ou pistas de contexto específico de uso para não ser descartado de início. Os critérios são os seguintes:

1) Critérios baseados no contexto: a) contexto determinativo - refere-se a palavras com significados específicos, facilmente identificáveis no contexto; b) identificação materna - envolve o conhecimento dos cuidadores em relação à produção da criança, de acordo com o pesquisador; c) uso múltiplo - quando a criança usa a mesma produção mais de uma vez; d) episódios múltiplos - observa-se a mesma produção com formas fonológicas semelhantes em diferentes contextos.

2) Critérios baseados no modelo de vocalização: a) correspondência complexa - a forma infantil corresponde a dois segmentos próximos à formaalvo; b) combinação exata - observa-se que até mesmo um ouvido não treinado consegue reconhecer um exemplo de palavra; c) correspondência prosódica - verifica-se uma correspondência prosódica (acento, quantidade de sílaba) da produção infantil com a forma alvo.

3) Relação com outras vocalizações: a) tokens imitados - observa-se que a criança compreende em sua produção o token imitado; b) invariante verifica-se todas as produções exibem as mesmas formas fonológicas; c) sem usos inadequados - as produções ocorrem em mesmo contexto de uso que sugerem o mesmo significado. (BAIA, 2013, p. 78-79)

Para Vihman e MacCune (1994), um bom candidato à palavra será aquele que apresentar pelo menos quatro critérios desses apresentados acima. Aquelas produções que apresentarem um número inferior serão categorizadas como balbucio. No Quadro 1, a seguir, são apresentados alguns exemplos dessas produções duvidosas de L. seguindo os critérios de Vihman e MacCune (1994): 
Quadro 1 - Exemplo de produções que geraram dúvidas de acordo com Vihman e McCune (1994)

\begin{tabular}{|l|l|c|c|c|c|c|c|c|c|c|c|c|}
\hline & Alvo & \multicolumn{4}{|c|}{ Contexto } & \multicolumn{3}{c|}{ Modelo vocal } & \multicolumn{3}{c|}{ Outro } & Decisão \\
\hline Candidato & & Determin & $\begin{array}{l}\text { M/P } \\
\text { id }\end{array}$ & $\begin{array}{l}\text { Uso } \\
\text { Mult. }\end{array}$ & $\begin{array}{l}\text { Epis. } \\
\text { Mult }\end{array}$ & Complexo & Exato & Prosod. & Imit. & $\begin{array}{l}\text { Invar. } \\
\begin{array}{l}\text { Sem } \\
\text { uso } \\
\text { inap. }\end{array}\end{array}$ \\
\hline [pa] & sapo & & $\mathrm{X}$ & & & & & & & & & NÃO \\
\hline ['na.na] & comida & $\mathrm{X}$ & $\mathrm{X}$ & $\mathrm{X}$ & & & & $\mathrm{X}$ & $\mathrm{X}$ & & $\mathrm{X}$ & SIM \\
\hline [du.'te] & Chulé & $\mathrm{X}$ & $\mathrm{X}$ & $\mathrm{X}$ & & & & $\mathrm{X}$ & $\mathrm{X}$ & & $\mathrm{X}$ & SIM \\
\hline ['ma.te] & menina & & $\mathrm{X}$ & & & $\mathrm{X}$ & & & & & & NÃO \\
\hline [ba.'ba] & Peppa & $\mathrm{X}$ & $\mathrm{X}$ & $\mathrm{X}$ & $\mathrm{X}$ & $\mathrm{X}$ & & $\mathrm{X}$ & $\mathrm{X}$ & & $\mathrm{X}$ & SIM \\
\hline
\end{tabular}

Outro ponto importante a ser considerado nessa categorização é a frequência. Ao categorizar as produções, leva-se em consideração tanto as palavras repetidas quanto as que foram produzidas uma única vez, i.e. consideram-se tokens e types, respectivamente.

Além disso, foram tabulados os dados, considerando as categorias fonológicas infantis. Vihman e MacCune (1994), a fim de determinar as categorias adequadas para descrever o uso das palavras produzidas pelas crianças, exploram um conjunto de contextos que acompanham a produção infantil:

1. Nominais: palavras referentes a animais, objetos e demais substantivos. Contexto: formas nominais usadas com referência a um conjunto de entidades, sugerindo a consciência da criança em relação ao tipo de token;

2. Relacionais: geralmente são interpretadas como palavras de evento, com mudanças temporais ou espaciais no ambiente, por exemplo, "atrás", "na frente".

3. Eventos: refere-se aos eventos realizados pelas crianças durante brincadeiras. Inclui palavras que marcam eventos simulados, como por exemplo, "alimentar a boneca", "dar chá para as amigas";

4. "Expressão social": palavras usadas para marcar interações sociais, por exemplo, "oi", "por favor".

5. Rotina/Jogo: palavras usadas como parte de rituais verbais ou rotinas não apoiadas por um contexto situacional mais amplo, incluindo sons de animais em resposta a questionamentos fora do contexto ( $\mathrm{baa}$ ) ou jogos como peeka-boo, "qual o tamanho do bebê?"etc.

6. Dêiticos: usadas para apontar pessoas, entidades ou eventos de interesse, por exemplo, "é esse". (VIHMAN; MACCUNE, 1994, p. 528, tradução nossa). ${ }^{7}$

\footnotetext{
7 "1. Nominals: words referring to animals, objects and other adult-noun referents.

Context-flexible: nominal forms used with reference to a range of entities, suggesting child awareness of typel token relationships.

Context-limited: nominal forms used in a limited way, to refer to a single referent or as part of a routinized context, such as labeling animals with their characteristic sounds while 'reading' with Mother. Specific: nominals used to refer to particular persons or entities. The category corresponds to the adult sub-class of proper nouns by the child (e.g. 'mommy', 'numnum' for a favorite blanke).

2 Relationals: words referring to reversible temporal or spatial transformations in the environment: 'allgone', 'back', 'more', 'up'. More than one use is required, to provide evidence of context-flexible application. Single uses of potential relational word are generally interpreted as 'event' words (which may be context-bound).

3. Event: used in relation to events which do not exhibit a reversible character. Includes words making pretend events (feeding doll-yum; rolling vehicle-vroom; serving tea-tea (to refer to a range of tea-
} 
Dentro desse repertório linguístico infantil, foram analisados os templates que são implementados pelas crianças, fenômeno que apresenta uma sistematização da entrada lexical no período inicial do desenvolvimento fonológico.

Durante as primeiras produções de palavras, muitas adaptações tendem a emergir na fala infantil. Em alguns casos, essas adaptações podem estar relacionadas com algum padrão específico, também chamado de template, i.e. rotinas articulatórias usadas de maneira sistemática para a expansão do léxico. Dessa maneira, os templates têm um impacto no desenvolvimento fonológico inicial, na medida em que servem como rotinas articulatórias que automatizam a aquisição das primeiras palavras e favorecem a expansão do léxico.

É importante ressaltar que não é qualquer padrão que deve ser considerado como template. Faz-se necessário verificar a frequência de ocorrência de padrões em um conjunto de palavras por sessões separadas. Buscando observar esses padrões, separadamente, no desenvolvimento de L., foi realizado um levantamento da frequência de templates.

O levantamento de templates foi realizado considerando duas frequências: a frequência de types e a frequência de tokens. Para esta, considerou-se uma frequência $\cong$ 40\%, conforme Baia (2013), e para aquela, 20\%, de acordo com Vihman e Croft (2007). Neste estudo, assumimos os templates emergentes considerando a frequência de tokens.

A categorização dos templates deu-se da seguinte maneira: foram observados todos os tokens produzidos por sessão e, em seguida, levantadas as estruturas fonológicas de palavras recorrentes nos dados de cada criança. Após isso, foi quantificado, em porcentagem, o total, separadamente, das estruturas emergentes nos dados infantis. A estrutura que apresentou um total de produção $\cong 40 \%$ tokens foi elencada como template.

Para ilustrar como essa emergência foi categorizada, é apresentado, a seguir, o Quadro 2, no qual é mostrada a distribuição dos templates recorrentes nos dados de $\mathrm{M}$. extraídos da tese de Baia (2013).

Quadro 2 - Distribuição dos templates nos dados de M. (BAIA, 2013, p. 186).

\begin{tabular}{|c|c|c|c|c|c|c|c|c|c|c|c|c|c|c|c|c|}
\hline & $0 ; 9$ & 0;10 & 0;11 & $1 ; 0$ & $1 ; 1$ & $1 ; 2$ & $1 ; 3$ & $1 ; 4$ & $1 ; 5$ & $1 ; 6$ & $1 ; 7$ & $1 ; 8$ & $1 ; 9$ & $1 ; 10$ & $1 ; 11$ & $2 ; 0$ \\
\hline $\mathbf{T}$ & & $\begin{array}{l}\text { C1V1.'C1V1 } \\
\text { C1V1.'C1V2 }\end{array}$ & $\begin{array}{l}\text { C1V1.'C1V1 } \\
\text { C1V1.'C1V2 }\end{array}$ & & & $\mathrm{CV}$ & CV & $\begin{array}{l}\text { C1V1.'C1V1 } \\
\text { C1V1.'C1V2 }\end{array}$ & & & & & & & & \\
\hline
\end{tabular}

O que não foi verificado como template, foi categorizado como fenômeno fonológico isolado. Isso remete às sessões em que não houve registro de um padrão operante com informações prosódicas e segmentais da palavra, mas foram observadas

related objects and actions); quaffing a drink-ah; sleeping-sh; cleaning-cleam) as well as real-life events (hurt finger-ow; sliding-whee).

4. Social expression: words used to mark (real or pretend) social interactions ('please', 'hi', 'yay').

5. Routine/game: words used as part of verbal rituals or routines not supported by a larger situational context, including animal sounds in response to questioning out of context ('baa'), or games such as 'peek-a-boo', 'how big is baby?' etc.

6. Deictic: words used to point out people, entities or events of interest ('this', 'that'), or to mark interest in general ('aha', 'look', 'oh')." 
tentativas de produção a partir de fenômenos fonológicos, como assimilação, apagamento etc.

\subsection{Característica da criança}

No Quadro 3, são apresentadas as características gerais da criança.

Quadro 3 - características gerais de L.

\begin{tabular}{|l|l|l|l|l|}
\hline Criança & \multicolumn{1}{|c|}{ Irmão } & \multicolumn{1}{|c|}{ Relação com os pais } & \multicolumn{1}{|c|}{ Socialização } & \multicolumn{1}{c|}{ Caráter } \\
\hline L. & Não tem & $\begin{array}{l}\text { Tem boa relação com os } \\
\text { pais }\end{array}$ & $\begin{array}{l}\text { Comunica e } \\
\text { dialoga bastante } \\
\text { com as pessoas } \\
\text { ao seu redor }\end{array}$ & $\begin{array}{l}\text { É desinibida } \\
\text { É determinada }\end{array}$ \\
\hline
\end{tabular}

Apresentadas essas características, apresentamos, a seguir, a hipótese a ser investigada.

\subsection{Hipótese}

Como já foi apresentado, a perspectiva dos SACs (THELEN; SMITH, 1994; LARSEN-FREEMAN, 1997; DE BOT et al., 2007) e o modelo dos exemplares (BYBEE, 2001，2008， 2016; CRISTÓFARO-SILVA, 2003) defendem que o desenvolvimento da linguagem é dependente da interação entre múltiplos elementos, e a experiência tem um impacto na representação fonológica.

Segundo Bybee (2016, p. 132), “[...] usar uma língua é uma questão de acessar representações estocadas, aquelas que são mais fortes [...] são acessadas mais facilmente e podem, então, ser mais facilmente usadas como base para a categorização de novos itens". Assim, os itens lexicais emergentes apresentam variabilidade ao longo do desenvolvimento fonológico inicial inter e intraindividual.

Com base nesses pressupostos, a seguinte hipótese é perseguida: como apresentado nas seções teóricas, o desenvolvimento fonológico não opera isoladamente, há uma estreita relação entre os padrões fônicos emergentes e outros aspectos presentes. Para abordar tal relação, faz-se necessário um modelo multirrepresentacional. Neste estudo, segue-se a perspectiva dos exemplares (BYBEE, 2001, 2002, 2008, 2016; CRISTÓFARO-SILVA; 2003; OLIVEIRA-GUIMARÃES, 2008;) no intuito de demonstrar tal relação e oferecer uma explicação alternativa para fenômenos como o PPP, que não parece ser possível explicar apenas nos limites fonológicos.

Na próxima seção, apresentamos a análise dos dados de L., tendo em vista a hipótese levantada.

\section{Análise e discussão dos dados}


O fenômeno PPP, segundo Macken (2008), caracteriza-se pela ocorrência de segmentos em um determinado grupo de palavras e por sua ausência em outro. No Quadro 4, são apresentados exemplos desse fenômeno, analisado a partir de tokens na fala de L. e da relação com os templates operantes. É importante salientar que este é o primeiro estudo que busca essa relação.

Quadro 4 - Ocorrência do fenômeno puzzle-puddle-pickle nos dados de L.

\begin{tabular}{|c|c|c|c|}
\hline Fenômeno puzzle-puddle-pickle & Frequência $^{8}$ & Faixa etária & $\begin{array}{c}\text { Total de } \\
\text { ocorrência do } \\
\text { fenômeno por } \\
\text { sessão } \\
\end{array}$ \\
\hline Não teve ocorrência do fenômeno & $\varnothing$ & $1 ; 0$ & $\varnothing^{9}$ \\
\hline $\begin{array}{l}\text { 'Renata' [da.'da] } \\
\text { 'Toma' [to.'mi] } \\
\text { 'beijo' [de] } \\
\text { 'abre' ['a.br] }\end{array}$ & $\begin{array}{l}{[\mathrm{t}] \rightarrow[\mathrm{d}]} \\
{[\mathrm{b}] \rightarrow[\mathrm{d}]}\end{array}$ & $\begin{array}{l}1 ; 1 \\
\text { Sem relação } \\
\text { com o template } \\
\text { operante } \\
\quad \mathbf{V}_{\text {médio-baixa }}\end{array}$ & 2 \\
\hline $\left.\begin{array}{l}\text { 'cocoricó' [to.to.'di] } \\
\text { 'brincar' ['bi.ke] } \\
\text { 'brinco' [mi.' go] } \\
\text { 'abrir' [a. 'bi] }] \\
\text { 'cuidado' [ta.'do] } \\
\text { 'dá' [da] } \\
\text { 'Fernanda' [na.'na] } \\
\text { 'chulé' [du.'da] }\end{array}\right\}$ & $\begin{array}{l}{[\mathrm{k}] \rightarrow[\mathrm{t}]} \\
{[\mathrm{b}] \rightarrow[\mathrm{m}] \quad(1)} \\
{[\mathrm{d}] \rightarrow[\mathrm{t}] \quad(2)} \\
{[\mathrm{d}] \rightarrow[\mathrm{n}] \quad(2)}\end{array}$ & $\begin{array}{l}\mathbf{1 ; 2} \\
\text { Sem template } \\
\text { operante }\end{array}$ & 4 \\
\hline Não teve ocorrência do fenômeno & $\varnothing$ & $1 ; 3$ & $\varnothing$ \\
\hline Não teve ocorrência do fenômeno & $\varnothing$ & $1 ; 4$ & $\varnothing$ \\
\hline $\begin{array}{l}\left.\begin{array}{l}\text { 'pintar' } \\
\text { 'pai' } \\
\text { [paj] }\end{array}\right] \\
\text { 'bater' ['baj.de] } \\
\text { 'gato' [to:] }\end{array}$ & $\begin{array}{l}{[\mathrm{p}] \rightarrow[\mathrm{d}](1)} \\
{[\mathrm{t}] \rightarrow[\mathrm{d}] \quad(1)}\end{array}$ & $\begin{array}{l}\mathbf{1 ; 5} \\
\text { Sem template } \\
\text { operante }\end{array}$ & 2 \\
\hline
\end{tabular}

\footnotetext{
${ }^{8}$ Essa frequência indica quantas vezes ocorreu a troca de um segmento pelo outro.

${ }^{9}$ Significa ausência de ocorrência do fenômeno PPP na sessão.
} 


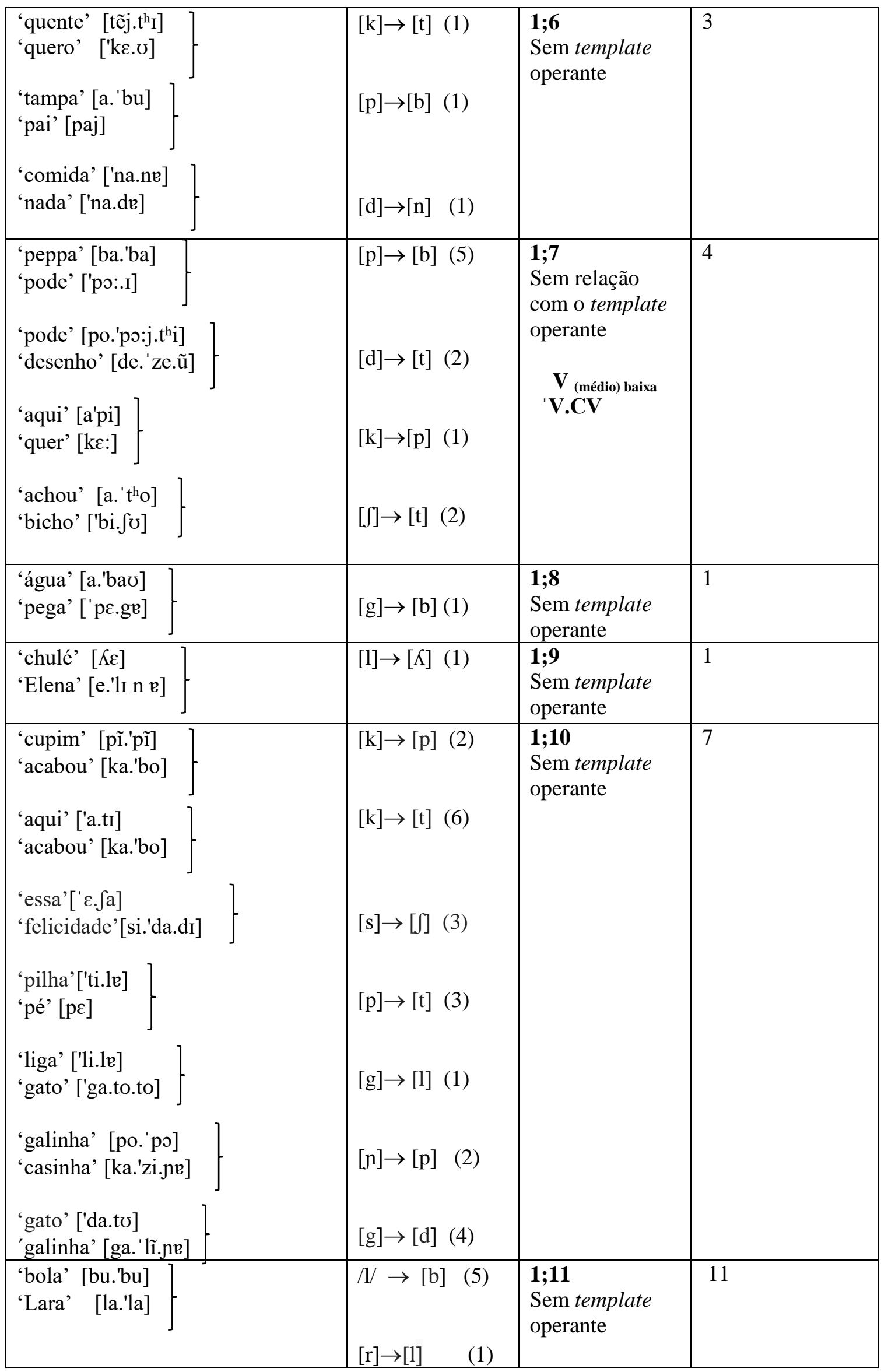




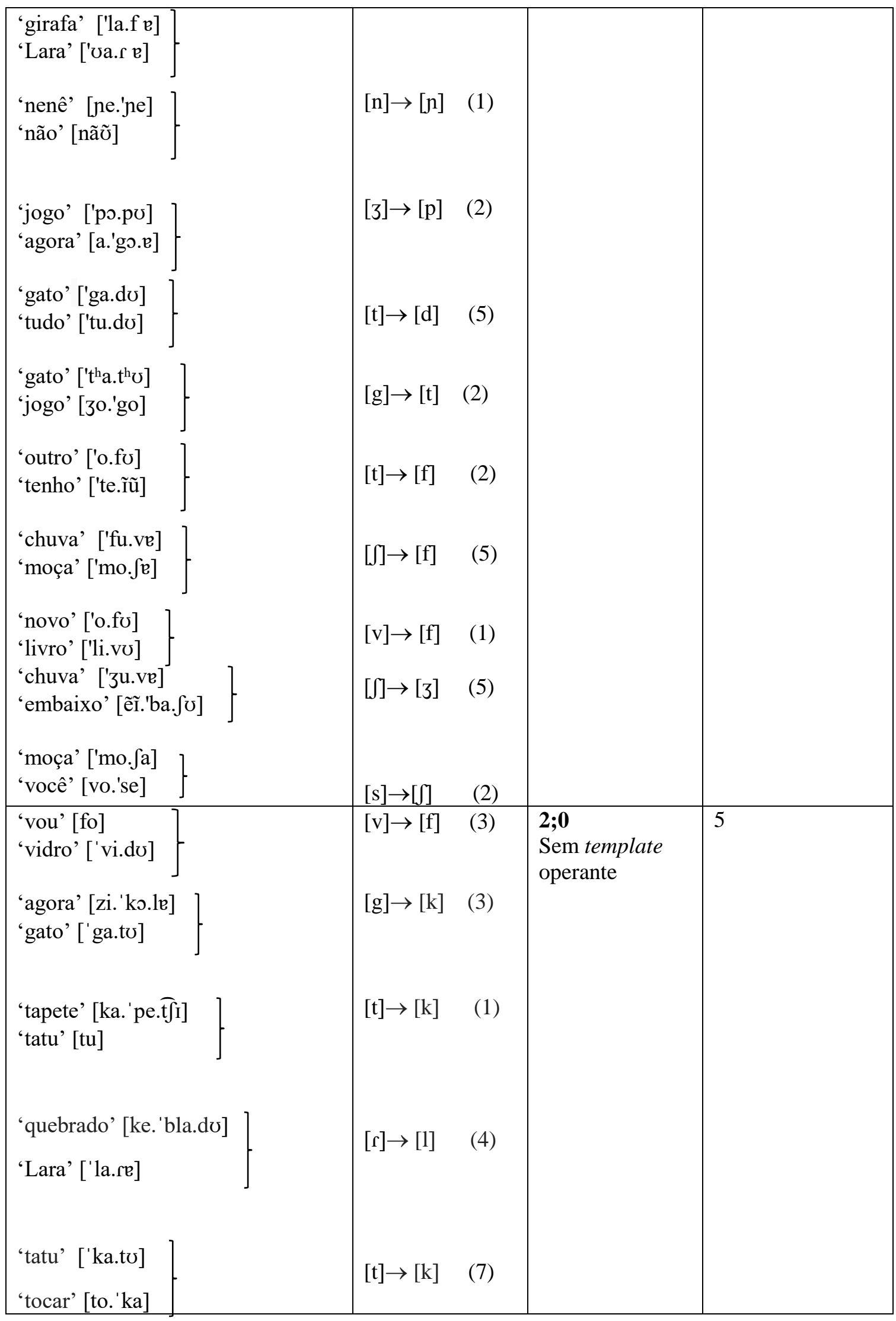


Com base no Quadro 4, é observada pouca ocorrência desse fenômeno nos dados de L. O quadro apresenta exemplos do fenômeno emergentes nos dados de L. ao longo de treze sessões. Por exemplo, na sessão 1;10, palavra "aqui" é produzida como ['a.tı], mas em um momento posterior, "acabou" é realizado como [ka.'bo].

Observamos, também, a falta de relação entre o fenômeno PPP e os templates operantes nas sessões em que houve indício de padrão sistemático. Um dos momentos é registrado na sessão 1;0, em que embora haja o template $\mathrm{V}_{\text {médio-baixa, o fenômeno não }}$ foi manifestado. O contrário ocorre na sessão 1;5, quando há registro do PPP e L. não faz uso de nenhum padrão específico. Interessantemente, na sessão 1;1, é registrada a emergência tanto de fenômeno quanto de template, no entanto não é verificada relação entre eles; é o caso da palavra "beijo" produzida como [de] e "abre" como ['a.bi], sendo o template operante $\mathrm{V}$.

É importante salientar que, nos dados de L., esse fenômeno teve influência do contexto prosódico, pois a maior parte das ocorrências foi em produções paroxítonas. A tabela 2 mostra os valores, em porcentagem, da posição da sílaba tônica nas palavras que apresentaram evidências desse fenômeno.

Tabela 2 - Posição tônica das palavras que evidenciaram o fenômeno $\mathrm{PPP}^{10}$

\begin{tabular}{ll}
\hline Posição da tônica & Porcentagem \\
\hline Oxítona & $33 \%(19)$ \\
Paroxítona & $77 \%(64)$ \\
\hline
\end{tabular}

Como pode ser visto na Tabela 2, o contexto preferível para que o fenômeno fosse manifestado foi em palavras paroxítonas, o que correspondeu a $77 \%$ (64) de produção, enquanto nas palavras oxítonas a emergência do fenômeno correspondeu a $33 \%$ (19).

Todavia, como já mencionado no início desta seção, em relação ao total de tokens produzidos em cada sessão, a ocorrência do fenômeno foi pouco frequente. A Tabela 3 mostra esses valores brutos e em porcentagem ao longo das sessões.

Tabela 3 - Relação entre a frequência total dos tokens e o fenômeno PPP

\begin{tabular}{llll}
\hline Sessão & Ocorrência do fenômeno & Frequência de tokens & Porcentagem \\
\hline $\mathbf{1 ; 0}$ & $\varnothing$ & 17 & $\varnothing^{11}$ \\
$\mathbf{1 ; 1}$ & 2 & 26 & $7,6 \%$ \\
$\mathbf{1 ; 2}$ & 6 & 82 & $7,3 \%$ \\
$\mathbf{1 ; 3}$ & $\varnothing$ & 18 & $\varnothing$ \\
$\mathbf{1 ; 4}$ & $\varnothing$ & 31 & $\varnothing$ \\
$\mathbf{1 ; 5}$ & 2 & 16 & $12,5 \%$ \\
$\mathbf{1 ; 6}$ & 3 & 57 & $5,2 \%$ \\
$\mathbf{1 ; 7}$ & 10 & 115 & $8,6 \%$ \\
$\mathbf{1 ; 8}$ & 1 & 72 & $1,3 \%$ \\
\hline
\end{tabular}

${ }^{10} \mathrm{O}$ total de produção foi contabilizado por sessão, considerando todas as produções de palavra que tenham manifestado o fenômeno (frequência de token). Ou seja, na troca de [1] $\rightarrow$ [b] em [bu.'bu] para "bola" ou [r] $\rightarrow$ [1] em [la.'la] para "Lara", foram consideradas todas as possibilidades de troca segmental.

${ }^{11}$ Não houve ocorrência do fenômeno. 


\begin{tabular}{llll}
\hline $\mathbf{1 ; 9}$ & 1 & 115 & $0,8 \%$ \\
$\mathbf{1 ; 1 0}$ & 22 & 296 & $7,4 \%$ \\
$\mathbf{1 ; 1 1}$ & 31 & 204 & $15,1 \%$ \\
$\mathbf{2 ; 0}$ & 18 & 206 & $8,7 \%$ \\
\hline
\end{tabular}

$\mathrm{Na}$ Tabela 3, apresentamos a relação da frequência de PPP com o total de tokens produzidos por L. Por meio dessa tabela, verificamos que apenas em duas sessões, especificamente nas sessões $1 ; 5$ e 1;11, essas ocorrências ultrapassaram 10\%. Nas demais sessões, isso não foi observado.

De acordo com o que foi apresentado e conforme a perspectiva holística, a emergência desse fenômeno é um desafio para estudos que partem de regras ordenadas/ armazenadas de maneira errônea (SMITH, 1963), ou para estudos que assumem haver um ordenamento específico e diferente das restrições da gramática adulta (DINNSENN; O'CONNOR; GIERUT, 2001), por estar relacionado especificamente a um grupo específico de palavras. Uma explicação alternativa seria a que apresentamos, que toma cada exemplar, palavra, de maneira isolada no desenvolvimento.

Dessa maneira, mantemos a hipótese de que o desenvolvimento fonológico não se desenvolve isoladamente, pois há uma estreita relação entre os padrões fônicos emergentes e outros aspectos presentes. Como, por exemplo, o fato de não haver relação entre os templates operantes e o PPP. Por essa razão, para abordar tal fenômeno, faz-se necessário um modelo multirrepresentacional (BYBEE, 2001, 2002, 2008, 2016; CRISTÓFARO-SILVA, 2003; OLIVEIRA-GUIMARÃES, 2008).

Destarte, podemos afirmar que o léxico não é produzido conforme o alvo de forma abrupta, mas por meio de instabilidade inicial. Essa instabilidade, pensando no fenômeno PPP, pode ser evidenciada por uma sobreposição de segmentos em diferentes representações de palavras. Além disso, considerando a perspectiva dos exemplares, o aumento do vocabulário de L. ao longo das sessões ao lado do aumento de ocorrência de PPPs podem estar relacionados à capacidade da criança em generalizar forma e significado de itens já experienciados.

Baseando-nos no modelo dos exemplares (BYBEE, 2001), podemos afirmar que cada item lexical acessado pela criança é armazenado em sua memória, permitindo uma representação múltipla e uma produção variável. Para tanto, a frequência é determinante nessa organização, pois possibilita, por meio da associação de itens já experienciados, a convencionalização automática do léxico emergente nos dados infantis.

Pensando nessa organização, podemos afirmar que o léxico gerencia a gramática (BYBEE, 2008). Esse léxico, conforme Bybee (2016, p. 174), "[...] consiste de forma e significado que é moldado em construções que são convencionalizadas, repetidas e sofrem mudança posterior tanto na forma como no significado". Associando a emergência lexical com os padrões emergentes, observamos que a frequência de um determinado padrão fônico permite que itens lexicais vão se tornando mais robustos ao serem mais produzidos, influenciando, por conseguinte, a organização fonológicolexical.

A emergência desses padrões, como os templates, em consonância com o modelo dos exemplares, é determinada pela frequência de uso que assume papel elementar na organização do sistema em desenvolvimento. Nessa perspectiva, defendemos que palavras e estruturas podem ganhar ou perder forças na memória conforme a frequência de uso. Cada vez que um padrão é acessado, a criança o 
armazena em sua memória. Dessa maneira, as representações linguísticas são estocadas a partir de generalizações ou de conexão entre as palavras que possuem padrões similares e que compartilham características semânticas em comum (BYBEE, 2001).

Podemos afirmar, ainda, que o léxico não é produzido conforme o alvo de forma abrupta, mas ele traça uma direção de mudança. Essas mudanças, pensando no fenômeno PPP, podem ser evidenciadas por uma sobreposição de exemplares.

Por fim, neste estudo, apresentamos resultados da análise do PPP considerando tokens, mas já está em andamento uma análise que parte da análise do mesmo fenômeno considerando types.

\section{Considerações finais}

Em linhas gerais, podemos afirmar que o fenômeno PPP, para ser explicado, precisa partir de um modelo que considere aspectos tanto fonológicos quanto lexicais, além de oferecer a possibilidade de analisar cada entrada lexical, cada token, de maneira independente, como o modelo dos exemplares permite. Além disso, por se tratar de um fenômeno não muito frequente, essa abordagem tem que estar aberta para investigar exceções, i.e o que é "caótico" no sentido de desviante, o que o paradigma dos SACs permite. Por fim, vale ressaltar que, conforme aponta Larsen-Freeman (1997), o estudo dos sistemas complexos e do caos - que é a aleatoriedade por eles manifestada - tem balançado as bases da ciência como um todo, dominada pelo pensamento linear e reducionista resultante dos trabalhos de Newton. Ao contrário da ideia de que a ciência é capaz de explicar o universo com exatidão, a descoberta da imprevisibilidade que acompanha sistemas maiores, mais complexos e não lineares põe em xeque uma concepção puramente determinista do universo e balança as bases do próprio fazer científico.

Este estudo segue aprofundando a análise do léxico inicial e sua relação com os diferentes componentes linguísticos e extralinguísticos na nuvem de exemplares.

\section{REFERÊNCIAS}

ALTOM, M. W.; MEDIN, D. L.; MURPHY, T. D. Given versus induced category representations: use of prototype and exemplar information in classification. Journal of experimental psychology: learning, memory and cognition, Illinois, v. 10, n. 3, p. 333$352,1984$.

BAIA, M. F. A. Os templates no desenvolvimento fonológico: o caso do português brasileiro. 2013. Tese (Doutorado em Linguística) - Faculdade de Filosofia, Letras e Ciências Humanas, USP, São Paulo, SP, 2013.

BOD, R.; COCHRAN, D. Introduction to Exemplar-Based Models of Language Acquisition and Use. St. Andrews (Escócia): School of Computer Science, University of St. Andrews, 2007. Disponível em: https://pdfs.semanticscholar.org/bda5/889b63d365a73d22b3b20432a842a4038839.pdf. Acesso em: 14 set. 2019. 
BRUM-DE-PAULA, M. R.; FERREIRA-GONÇALVES, G. Léxico \& gramática: uma relação de causa e efeito? Letras de Hoje, Porto Alegre, v. 43, n. 3, p. 69-80, 2008.

BYBEE, J.; CACOULLOS, T. Phonological and Grammatical Variation in Exemplar Models. Studies in Hispanic and Lusophone Linguistics, New Mexico, v. 1, p. 399-413 2008.

BYBEE, J. Phonology and Language Use. Cambridge: Cambridge University Press, 2001.

Phonological evidence for exemplar storage of multiword sequences. Studies in Second Language Acquisition, Cambridge, v. 24, n. 2, p. 215-221, 2002.

Usage-based grammar and second language acquisition. In: ROBINSON, P.; ELLIS, N. C. Handbook of cognitive linguistics and second language acquisition. New York: Routledge, 2008. p. 216-236.

.Usage-based theory and exemplar representations of constructions. In:

HOFFMANN, Thomas. The Oxford Handbook of Construction Grammar. Oxford: Oxford University Press, 2013. p.49-69.

Língua, uso e cognição. Tradução de Maria Angélica Furtado da Cunha. São Paulo: Cortez, 2016.

CRISTÓFARO-SILVA, T. Descartando fonema: a representação mental na fonologia de uso. Minas Gerais. In: DA HORA, D; COLLISCHON, G. Teoria Linguística: fonologia e outros temas. João Pessoa: Editora Universitária/UFPB, 2003. p. 200-231

CRISTÓFARO-SILVA, T.; OLIVEIRA-GUIMARÃES, D. M. L. A aquisição de africadas alveopalatais: contribuições teóricas e metodológicas. In: FERREIRAGONÇALVES, G.; BRUM-DE-PAULA, M. R.; KESKE-SOARES, M. (Org.). Estudos em Aquisição fonológica. Pelotas: Editora e Gráfica Universitária-UFPel, 2011. p. 3146.

DE BOT, K. et al. A Dynamic Systems Theory approach to second language acquisition. Bilingualism: Language and Cognition, Groningen, v. 10, n. 1, p. 7-21, 2007.

DINNSEN, D. A.; O'CONNOR, K. M.; GIERUT, J. A. The puzzle-puddle-pickle problem and the Duke-of-the-York gambit in acquisition. Journal of Linguistics, Cambridge, v. 37, n. 3, p. 503-525, 2001.

GERKEN, L. Language development. Arizona: Plural Publishing, 2008.

JAKOBSON, Roman. Child language, aphasia and phonological universals. Paris: Mouton, 1972 [1941]. 
LAMPRECHT, R. R. et al. Aquisição fonológica do português: perfil de desenvolvimento e subsídios para terapia. Porto Alegre: Artmed, 2004.

LANGACKER, R. W. A Dynamic Usage-Based Model. In: BARLOW, M.; KEMMER, S. (Org.). Usage-Based Model of Language. Stanford: CSLI Publications, 2000.

LARSEN-FREEMAN, D .Chaos/Complexity Science and second language acquisition. Applied Linguistics, Oxford, v. 18, n. 2, p. 141-165, 1997.

LEVELT, C. C. et al. The acquisition of syllable types. Language Acquisition, Nijmejen, v. 8, n. 3, p. 237-264, 2000.

MACKEN, M. A. The child's lexical representation: the 'puzzle-puddle-pickle' evidence. Journal of Linguistics, Cambridge, v. 16, p. 1-7, 2008 [1980].

MATLIN, M. Psicologia Cognitiva. Rio de Janeiro: LTC, 2004.

MCCARTHY, J.; PRINCE, A. Prosodic Morphology: constraint interaction and satisfaction. Report no. RuCCS-TS-3. New Brunswick: Rutgers University Center for Cognitive Science, 1993.

OLIVEIRA-GUIMARÃES, D. M. L. Percurso de construção da fonologia pela criança: uma abordagem dinâmica. 2008. Tese (Doutorado em Linguística) - Faculdade de Letras, UFMG, Belo Horizonte, MG, 2008.

PIERREHUMBERT, J. Exemplar dynamics: word frequency, lenition and contrast. In: BYBEE, J. L.; HOPPER, P. (Org.). Frequency and the Emergency of Linguistic Structure. Amsterdam: John Benjamins, 2003. p. 137-157.

. The dynamic lexicon. In: COHN, A.; HUFFMAN, M.; FOUGERON, C. (Org.). Handbook of Laboratory Phonology. Oxford: Oxford University Press, 2012. p. 173-183.

SCHWINDT, L. C. Teoria da Otimidade e fonologia. In: BISOL, L. Introdução aos estudos de fonologia do português brasileiro. 5a. ed. Porto Alegre: Edipucrs, 2010. p. 236-258.

SMITH, M. The exemplar View. In: Categories and Concepts. Cambridge: Harvard Press, 1981. p.143-161.

SMITH, N. The acquisition of Phonology. Cambridge: Cambridge University Press, 1963.

THELEN, E.; SMITH, L. B. A Dynamic Systems Approach to the Development of Cognition and Action. Cambridge: MIT Press, 1994.

VIGÁRIO, M.; FROTA, S.; MARTINS, F. A frequência que conta na aquisição da fonologia: types ou tokens. In: BRITO, A. M.; SILVA, F.; VELOSO, J.; FIÉIS, A. 
(Org.). XXV Encontro Nacional da Associação Portuguesa de Linguística. Porto: Associação Portuguesa de Linguística, 2010. p. 749-767.

VIHMAN, M. M. Variable paths to early word production. Journal of Phonetics, Cidade, v. 21, p. 61-82, 1993.

VIHMAN, M. M.; CROFT, W. Phonological development toward a "radical" templatic phonology. Linguistics, Berlin, v. 45 n. 4, p. 683-725, 2007.

VIHMAN, M. M.; MCCUNE, L. When is a word a word? Journal of Child Language, Cambridge, v. 21, p. 517-542, 1994.

WIETHAN, F. M. et al. O paradigma conexionista aplicado às pesquisas em linguagem. Cefac, São Paulo, v. 14, n. 5, p. 984-991, 2012. 